\title{
ON ANALYTICAL INVESTIGATION AND SIMULATION OF HM-NETWORKS WITH PRIORITY REQUESTS
}

\author{
Natalia Bekish ${ }^{1}$, Olga Kiturko ${ }^{1}$, Mikhail Matalytski $^{2}$ \\ ${ }^{I}$ Faculty of Mathematics and Computer Science, Grodno State University, Belarus \\ ${ }^{2}$ Institute of Mathematics, Czestochowa University of Technology, Poland \\ nat67@list.ru,sytaya_om@mail.ru,m.matalytski@gmail.com
}

\begin{abstract}
This paper describes finding the expected incomes HM (Howard-Matalytski) networks with priority requests both analytically and through simulation. The analytical results for the study of such networks can be used as stochastic models for the prediction of income of enterprises and banking information networks, logistics systems, transportation and other facilities. The basic principles of simulation networks using discrete-event simulation, using the open software library SimPy (Simulation in Python) package of Python. This library allows for the simulation of different processes in parallel, and describes the logic of interaction between processes. The application of simulation for solving the problems of finding the expected incomes the logistics transport system is considered.
\end{abstract}

\section{Introduction}

Queuing systems (QS) networks are models of systems in which requests need of service come to some serving device. Examples include user requests received from the server computer network, the aircraft arriving at the airport, ships calling at the port and cars and trains arriving at loading or unloading. Under the QS network refers to a set of queuing systems which circulates requests, moving in accordance with the matrix of transition probabilities from one system to another.

The analytical study and simulation are the main methods of research QS networks. However, simply formulated mathematical models of queuing theory do not always lend themselves to simple mathematical analysis. Most occur in the study of network problems that can not be solved by using the known exact methods of network theory. One of the main reasons for this is that moving between streams in the network applications are of complex statistical dependence. This is especially true of QS networks, and service times of applications in systems which are distributed by the laws of a non-exponential. In this situation, using simulation.

The process of simulation consists of the fact that the play operation of the network queuing on a PC using the appropriate algorithms and simulation experiments are carried out; calculated characteristics of the network. Involvement of simulation is explained by the fact that it is possible with the help of numerical experiments on a PC to confirm the approximate analytical calculations with 
respect to those or other characteristics of the studied QS networks. In addition, the advantage and the dignity of modelling is that it applies to systems and networks of any complexity, where an analytical study is very difficult or even impossible.

Simulation models are designed for:

- statistical estimation of parameters characterizing the operation of QS networks;

- verification of statistical hypotheses concerning the parameters of these QS networks;

- verification of the accuracy of the numerical analysis of QS networks, etc.

Consider HM (Howard-Matalytski) - a network of arbitrary structure with the same type of applications, consisting of $n$ queuing systems (QS) $S_{1}, S_{2}, \ldots, S_{n}$. The request for passing from one to other QS brings the latter system income and profit respectively in the first system and is reduced by this amount. HM-networks are used as models to predict earnings in banking, information networks, logistic transport systems and other objects [1].

The most common type of simulation is a discrete event simulation. It is a simulation of the system at discrete points in time when events occur that reflect the changing state of the system over time. In dynamic systems, which we consider, the state of the system, properties - it is a function of time, which in the modelling process is constantly changing.

The active-oriented model, event-based model and process-oriented model are part of a discrete-event simulation. This article describes the general principles of modelling HM-networks with priority requests with the library SimPy [2].

\section{Analytical investigation of HM-networks with priority requests}

Consider a closed network in which circulates $K_{1}$ requests of the first type and $K_{2}$ requests of the second type, and requests that can not change its type. The matrix of transition probabilities between the systems network requests $\left\|p_{i j}\right\|_{n \times n}$ is irreducible. System $S_{i}$ contains $m_{i}$ parallel lines of service, the service time requests of each type every line of the system has an exponential distribution, $i=\overline{1, n}$. The probability of service requests type $c$ in the lines of $S_{i}$ during the time interval $[t, t+\Delta t]$ is $\mu_{i c}(t) \Delta t+o(\Delta t)$, where $\mu_{i c}(t)$ - intensity of service requests such as $c$ in every line system $S_{i}$ at time $t, i=\overline{1, n}, c=1,2$. Functions $\mu_{i c}(t)$ will be considered restricted for each time interval, $i=\overline{1, n}, c=1,2$.

The same type of request in the queue of some queuing system (QS) are selected for service in random order, for example, FIFO. Requests of the first type have absolute priority over the requests of the second type. In this case it means the fulfilment of two conditions: a) if at the time of the release of a QS line after the service requests in its queue has priority requests, then any of them may occupy 
the vacant line, and b) if the service system, all lines which are busy, but not only the priority of applications received priority application, it replaces the lowpriority request from one of the lines and beains to service this line, the request is extruded into consideration all the QS. When the request goes to the repressed service again, it served over the remaining service time. Since the service time is exponentially distributed, we can assume that the displacement re-application will be maintained, ie we have the so-called non-identical service.

Network states in this case given by the vector $k(t)=(k, t)=$ $=\left(k_{11}, k_{12} ; k_{21}, k_{22} ; \ldots ; k_{n 1}, k_{n 2} ; t\right)$, where $k_{i c}$ - number of requests of the type $c$ in QS $i, \sum_{i=1}^{n} k_{i 1}=K_{1}, \sum_{i=1}^{n} k_{i 2}=K_{2}$.

Let $I_{i 1}$ - vector of dimension $2 n$ with zero components, behind an exception component with number $2 i-1$, which is equal $1, I_{i 2}$ - vector of dimension $2 n$ with zero components, behind an exception component with number $2 i$, which is equal $1, I_{0}$ - vector of dimension $2 n$ with zero components

$$
\begin{aligned}
& k+I_{i 1}-I_{j 1}=\left(k_{11}, k_{12} ; \ldots ; k_{i 1}+1, k_{i 2} ; \ldots ; k_{j 1}-1, k_{j 2} ; \ldots ; k_{n 1}, k_{n 2}\right), \\
& k+I_{i 2}-I_{j 2}=\left(k_{11}, k_{12} ; \ldots ; k_{i 1}, k_{i 2}+1 ; \ldots ; k_{j 1}, k_{j 2}-1 ; \ldots ; k_{n 1}, k_{n 2}\right) .
\end{aligned}
$$

The system of equations for the state probabilities $P(k, t)$ has the form:

$$
\begin{aligned}
& \frac{d P(k, t)}{d t}=-\sum_{\substack{i, j=1 \\
i \neq j}}^{n}\left[\mu_{i 1}(t) \varepsilon_{i 1}\left(k_{i 1}\right)+\mu_{i 2}(t) \varepsilon_{i 2}\left(k_{i 1}, k_{i 2}\right)\right] p_{i j} P(k, t)+ \\
& +\sum_{\substack{i, j=1 \\
i \neq j}}^{n}\left[\beta_{i 1}(k, t) P\left(k+I_{i 1}-I_{j 1}, t\right)+\gamma_{i j}(k, t) P\left(k+I_{i 2}-I_{j 2}, t\right)\right],
\end{aligned}
$$

where

$$
\begin{gathered}
\beta_{i j}(k, t)=\mu_{i 1}(t) \varepsilon_{i 1}\left(k_{i 1}\right) u\left(k_{i 1}\right) u\left(K_{1}-k_{j 1}\right) p_{i j}, \\
\gamma_{i j}(k, t)=\mu_{i 2}(t) \varepsilon_{i 2}\left(k_{i 1}, k_{i 2}\right) u\left(k_{i 2}\right) u\left(K_{2}-k_{j 2}\right) p_{i j}, \\
\varepsilon_{i 1}\left(k_{i 1}\right)=\min \left\{k_{i 1}, m_{i}\right\}, i=\overline{1, n}, \\
\varepsilon_{i 2}\left(k_{i 1}, k_{i 2}\right)=\left\{\begin{array}{l}
k_{i 2}, k_{i 1}+k_{i 2}<m_{i}, \\
m_{i}-k_{i 1}, k_{i 1}<m_{i}, k_{i 1}+k_{i 2} \geq m_{i}, i=\overline{1, n}, \\
0, k_{i 1} \geq m_{i} .
\end{array}\right.
\end{gathered}
$$

It displayed the same way as in the case when the intensity of service requests in the lines of systems does not depend on time [1]. 
Let's designate through $v_{i}(k, t)$ - the full expected income which is received by system $S_{i}$ at time $t$, if during the initial moment of time the network is in state $k$; $r_{i}(k, t)$ - the income of system $S_{i}$ in unit of time, when the network is in state $(k, t) ; r^{(1)}\left(k+I_{i 1}-I_{j 1}, t\right)$ - the income of system $S_{i}$ (the expense or loss of system $\left.S_{j}\right)$, when the network changes state from $(k, t)$ to $\left(k+I_{i 1}-I_{j 1}, t+\Delta t\right)$ during time $\Delta t, r^{(2)}\left(k+I_{i 2}-I_{j 2}, t\right)$ - the income of system $S_{i}$ (the expense or loss of system $\left.S_{j}\right)$, when the network changes state from $(k, t)$ to $\left(k+I_{i 2}-I_{j 2}, t+\Delta t\right)$ during time $\Delta t, j=\overline{1, n}, j \neq i$.

During the time interval $\Delta t$ the network may remain in state $(k, t)$ or change its state on $\quad\left(k+I_{i 1}-I_{j 1}, t+\Delta t\right), \quad\left(k-I_{i 1}+I_{j 1}, t+\Delta t\right), \quad\left(k+I_{i 2}-I_{j 2}, t+\Delta t\right)$, $\left(k-I_{i 2}+I_{j 2}, t+\Delta t\right)$. If during $\Delta t$ the network passes to state $\left(k+I_{i 1}-I_{j 1}, t+\Delta t\right)$ with probability $\beta_{j i}\left(k+I_{i 1}-I_{j 1}, t\right)=\mu_{j 1}(t) \varepsilon_{j 1}\left(k_{j 1}\right) u\left(k_{j 1}\right) u\left(K_{1}-k_{i 1}\right) p_{j i} \Delta t+o(\Delta t)$, the income of system $S_{i}$ is equal $r^{(1)}\left(k+I_{i 1}-I_{j 1}, t\right)$ plus the expected income $v_{i}\left(k+I_{i 1}-I_{j 1}, t\right)$ of the network over the remaining time under the assumption that the initial network state was $\left(k+I_{i 1}-I_{j 1}\right), j=\overline{1, n}$. If during $\Delta t$ the network passes to state $\left(k+I_{i 2}-I_{j 2}, t+\Delta t\right)$ with probability $\gamma_{j i}\left(k+I_{i 2}-I_{j 2}, t\right)=$ $=\mu_{j 2}(t) \varepsilon_{j 2}\left(k_{j 1}, k_{j 2}\right) u\left(k_{j 2}\right) u\left(K_{2}-k_{i 2}\right) p_{j i} \Delta t+o(\Delta t)$, the income is equal $r^{(2)}\left(k+I_{i 2}-I_{j 2}, t\right)$ plus the expected income of the network over the remaining time under the assumption that the initial network state was $\left(k+I_{i 2}-I_{j 2}\right), j \neq i$. If during $\Delta t$ the network passes to state $\left(k-I_{i 1}+I_{j 1}, t+\Delta t\right)$ with probability $\beta_{i j}\left(k-I_{i 1}+I_{j 1}, t\right)=\mu_{i 1}(t) \varepsilon_{i 1}\left(k_{i 1}\right) u\left(k_{i 1}\right) u\left(K_{1}-k_{j 1}\right) p_{i j} \Delta t+o(\Delta t)$, the income of system $S_{i}$ is equal $r^{(1)}\left(k-I_{i 1}+I_{j 1}, t\right)$ plus the expected income $v_{i}\left(k-I_{i 1}+I_{j 1}, t\right)$ over the remaining time under the assumption that the initial network state was $\left(k-I_{i 1}+I_{j 1}\right), \quad j=\overline{1, n}$. If during $\Delta t$ the network passes to state $\left(k-I_{i 2}+I_{j 2}, t+\Delta t\right)$ with probability $\gamma_{j i}\left(k-I_{i 2}+I_{j 2}, t\right)=$ $=\mu_{i 2}(t) \varepsilon_{i 2}\left(k_{i 1}, k_{i 2}\right) u\left(k_{i 2}\right) u\left(K_{2}-k_{j 2}\right) p_{i j} \Delta t+o(\Delta t), \quad$ the income equal $r^{(2)}\left(k-I_{i 2}+I_{j 2}, t\right) \quad$ plus the expected income over the remaining time under the assumption that the initial the network state was $\left(k-I_{i 2}+I_{j 2}\right), j \neq i$. Similarly, if the network remains in state $(k, t+\Delta t)$ with probability $1-\sum_{\substack{j=1, j \neq i}}^{n}\left[\left(\mu_{j 1}(t) \varepsilon_{j 1}\left(k_{j 1}\right)+\right.\right.$ 
$\left.\left.+\mu_{j 2}(t) \varepsilon_{j 2}\left(k_{j 1}, k_{j 2}\right)\right) p_{j i}+\left(\mu_{i 1}(t) \varepsilon_{i 1}\left(k_{i 1}\right)+\mu_{i 2}(t) \varepsilon_{i 2}\left(k_{i 1}, k_{i 2}\right)\right) p_{i j}\right] \Delta t+o(\Delta t)$, the income of system $S_{i}$ is equal $r_{i}(k, t) \Delta t+v_{i}(k, t)$.

Then, using the total probability formula for conditional expectation, we can obtain a system of difference-differential equations (DDE), which in the matrix form can be rewritten as

$$
\frac{d V_{i}(t)}{d t}=Q_{i}(t)+A_{i}(t) V_{i}(t), i=\overline{1, n}
$$

where $V_{i}^{T}(t)=\left(v_{i}(1, t), \ldots, v_{i}(L, t)\right)$ - vector of incomes of the system $S_{i}, \quad L$ - the number of network states.

The number of priority and non-priority requests do not depend on each other, so the number of states in the considered network equals the number of ways to place the priority requests $K_{1}$ and non-priority requests $K_{2}$ to service systems, that is $L=C_{n+K_{1}-1}^{n-1} C_{n+K_{2}-1}^{n-1}$.

\section{Simulation of HM-networks with priority requests}

The process-oriented model is used to simulate the HM-networks. It is based on the use of objects of two types: 1) the active type - processes, 2) non-active types resources. Processes (active objects) include the application served on the network that correspond to reality, and the resources include a variety of queuing networks.

We construct a simulation model of changes in income of systems with closed HM-network with priority requests. We consider the objects of these two types. Each resource has a set of properties. These properties include the servicing capacity, which equals the number of service lines in the QS, and the distribution of service times of requests in each line. For each process a priority is given, and is defined as follows: either the request has priority (the first type of requests) in the service or not (the second type of requests). Processes with priority are served first, and processes without priority must wait.

Since the processes are active objects, they can "grab" resources when they are free, "hold" them for some time, or stand in line to resources, which is equivalent to service requests in the queuing system. After the capture and retention of a single resource, the process proceeds to a different resource, according to the matrix of transition probabilities. During the transition from one resource to another, a certain income moves from resource to resource. This value is determined by the generator, the associated resources involved in the transition.

It is necessary to consider the objects of another type, called a generators of income. Random variables $R_{i j}, r_{i}, i=\overline{1, n}$ are reimages of the generators of income in the model. In a simulation model the proceeds of transitions between states of 
the network may be random. Each QS should be compared to the three generators of income - the first to get the value of the gains from the transition from the requests of the first type of the QS to another, first to get the value of the gains from the transition from the requests of the first type of the QS to another and the third for winning the QS over the time interval during which all bids were at the service.

We used the package SimPy (Simulation in Python) - an open software library for simulation of different processes with discrete events, written in the Python programming language. This fully object-oriented library provides developers with all the necessary tools to develop simulation models of almost any complexity. It allows for the simulation of different processes in parallel, and also allows us to describe the logic of interaction between processes. To present the simulation components in the package, there are two main classes - Process for the active entities, processes, groups of entities shared processes and Resource - for resources. In addition, introduced an important class of Monitor, which collects the simulated data for analysis and statistics.

Example 1. Consider a closed Markov HM-network with the priority requests, consisting of $7 \mathrm{QS}, n=5, S_{1}, \ldots, S_{6}$ - peripherals, $S_{7}$ - the central. Let $m_{i}$ - number of service lines in the system $S_{i}, m_{1}=2, m_{2}=1, m_{3}=3, m_{4}=1$, $m_{5}=2, m_{6}=8, m_{7}=1$; transition probabilities of requests between the QS have the form: $p_{i n}=1, p_{n i}=2 /(3 n+1), i=\overline{1, n}$, all other transition probabilities are equal to 0 . In the network 8 requests of the first type are circulating, $K_{1}=8$, and 6 requests of the second type, $K_{2}=6$. The service times of requests in the peripheral systems of the network are distributed exponentially with parameter 2 , and in the central system network - by the normal distribution with mean 1 and variance 2.

Let us consider the case where the proceeds of transitions between states of the network are random variables $r^{(1)}, r^{(2)}$, uniformly distributed on $[1 ; 10]$. And let the values $r_{i}$ are constant and take the values $r_{i}=3-2 i, i=\overline{1, n}$. Suppose also that at the initial time in all the QS network incomes are equal to zero.

Using simulation, the values of expected income systems on the network are obtained. The schedule of income of the central QS is shown in Figure 1.

Consider the model of logistic transportation system diagram is shown in Figure 2. A model of this system can act HM-network of 17 QS $S_{1}, S_{2}, \ldots, S_{17}$. [2] Requests on the network are trains, moving from one station to another (system service).

The number of service lines in each system is given by the vector $m=(2,5,1,2,7,3,8,3,3,2,6,1,2,5,1,2,5) . \quad 100$ requests the first type 200 requests of the second type are circulated in the network, $K_{1}=100$, $K_{2}=200$. 


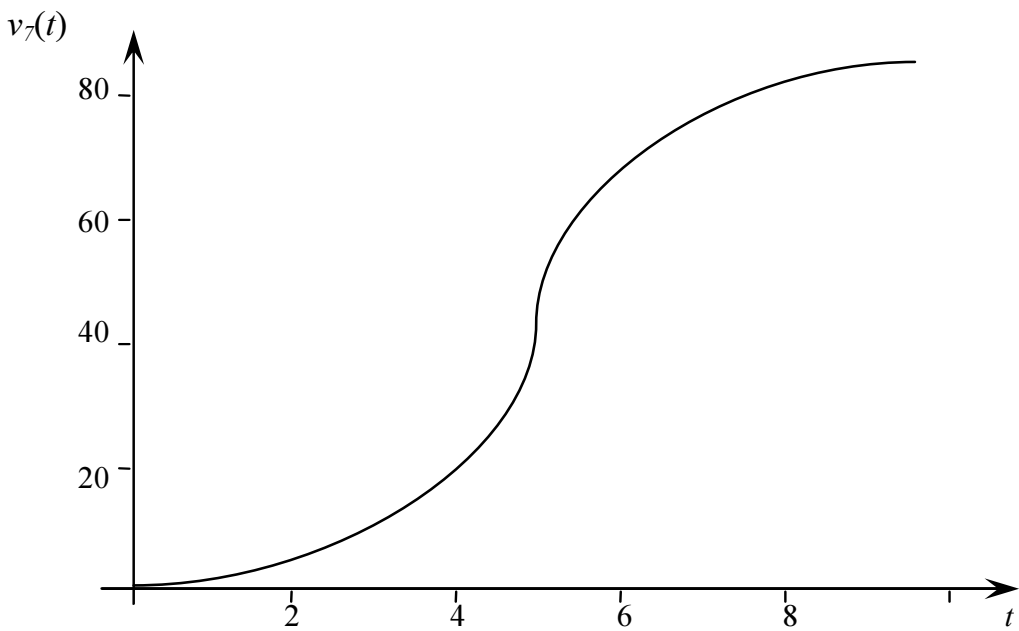

Fig. 1. Schedule of anticipated income for the central system

Let the service times requests in the systems, respectively, exponentially distributed with parameters: $\mu_{1}=\mu_{7}=\mu_{10}=\mu_{13}=\mu_{14}=1, \quad \mu_{2}=4$, $\mu_{3}=\mu_{9}=\mu_{11}=\mu_{12}=\mu_{15}=3, \mu_{4}=\mu_{8}=\mu_{16}=1, \mu_{5}=5, \mu_{6}=\mu_{17}=6$.

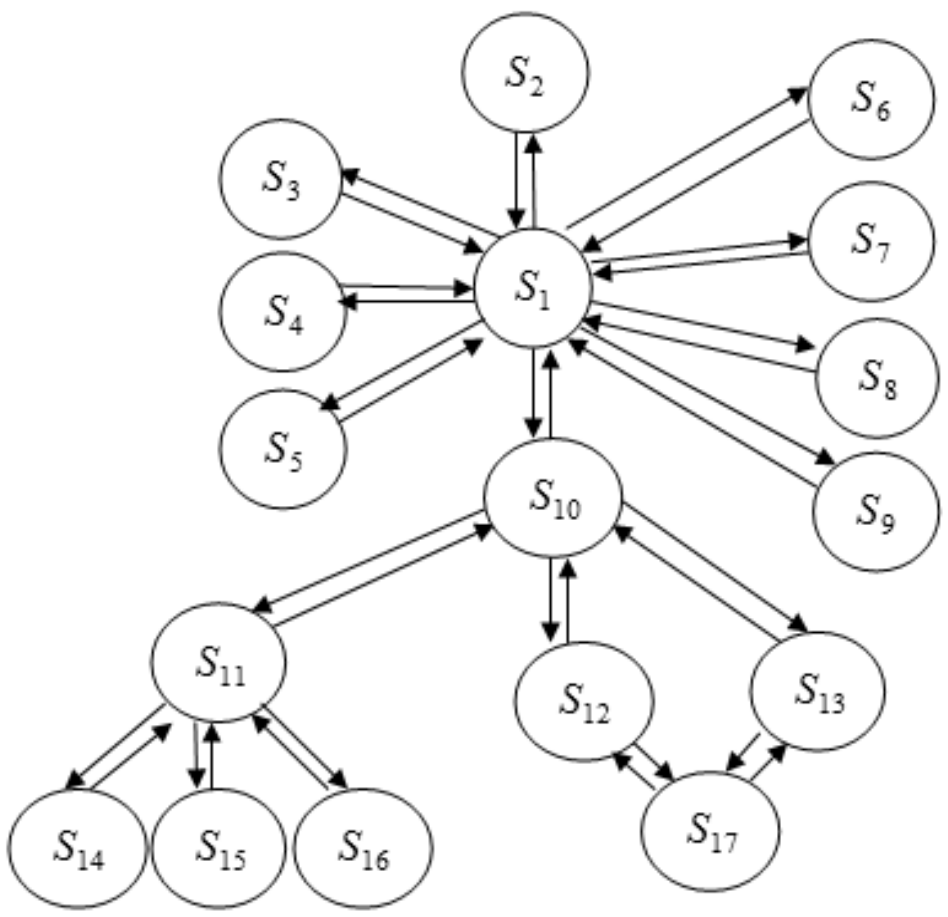

Fig. 2. Scheme of the considered network 
Let us consider the case where the proceeds of transitions between states of the network $r^{(1)}, r^{(2)}$ are random variables, uniformly distributed on the interval $[50 ; 100]$. And let the value be constant and take values $r_{i}=5-i, i=\overline{1, n}$. Suppose also that at the initial time income in network systems are equal to zero.

Using the simulation results are as follows (Figure 3).

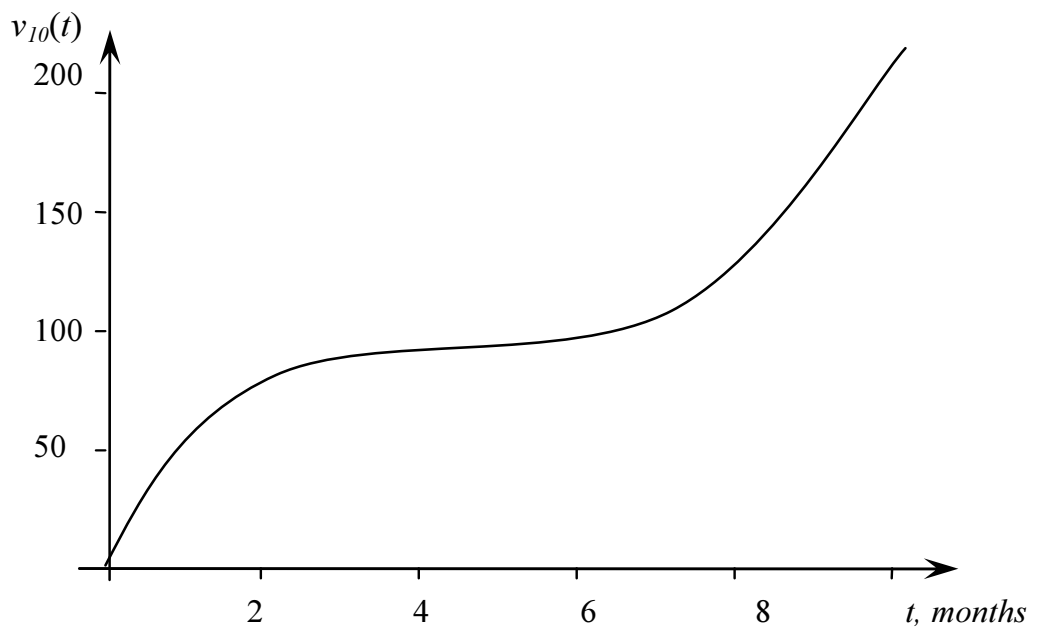

Fig. 3. Schedule of income tenth of the network

Example 2. Consider a network model of the fragment shown in Figure 4.

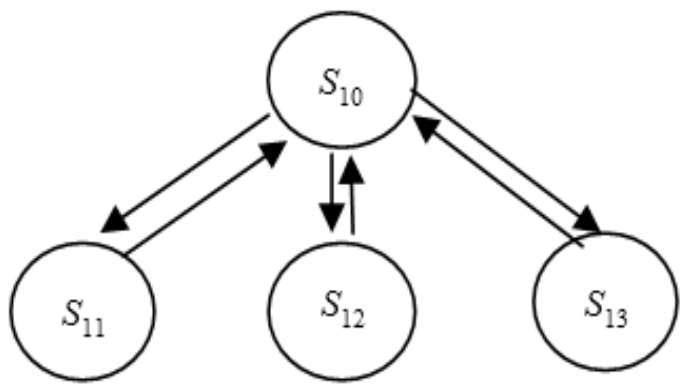

Fig. 4. Grodno transport logistics system

Let the matrix of transition probabilities between the systems is as follows:

$$
P=\left\|p_{i j}\right\|_{4 \times 4}=\left(\begin{array}{cccc}
0 & 0 & 0 & 1 \\
0 & 0 & 0 & 1 \\
0 & 0 & 0 & 1 \\
0.5 & 0.25 & 0.25 & 0
\end{array}\right) .
$$


The intensity of service requests are defined as follows: $\mu_{1}(t)=\sin (3 t+1)$, $\mu_{2}(t)=2 t+2, \mu_{3}(t)=2-\sin 4 t, \mu_{4}(t)=5 t+1$, and the service times of applications in the systems are exponentially distributed with parameter 0.8 .

In the network, 10 requests the first type and 5 applications of the second type circulate, $K_{1}=10, K_{2}=5$. The matrices of the proceeds of the transitions between the QS systems have the form:

$$
r^{(1)}=\left(\begin{array}{cccc}
0 & 0 & 0 & 0 \\
0 & 0 & 0 & 0 \\
0 & 0 & 0 & 0 \\
30 & 400 & 380 & 0
\end{array}\right), r^{(2)}=\left(\begin{array}{cccc}
0 & 0 & 0 & 0 \\
0 & 0 & 0 & 0 \\
0 & 0 & 0 & 0 \\
20 & 50 & 70 & 0
\end{array}\right) .
$$

Suppose also that at the initial time income networks for all systems are equal to zero.

Using the simulation results are as follows (Figure 5).

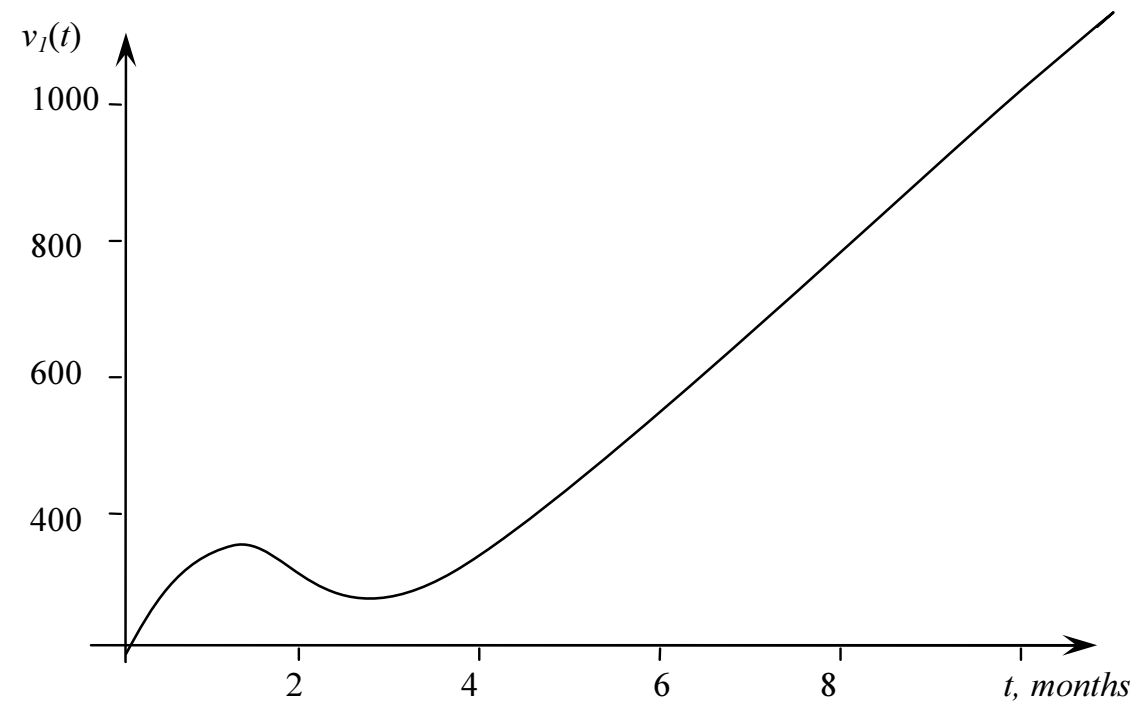

Fig. 5. Income of system $S_{1}$ for initial state of network

\section{Conclusions}

So, we have presented the system of equations for the expected income and described the basic principles of simulation HM-networks with priority requests using discrete-event simulation, using an open software library SimPy (Simulation in 
Python) package of Python. This library allows for simulating various parallel processes, and allows for describing the logic of interaction between processes. We also consider the use of simulation for determining the expected revenue models specific logistics of the transport system.

\section{References}

[1] Matalytski M., Tixonenko O., Koluzaeva K., Systems and queuing networks: analysis and application, GrSU, Grodno 2011.

[2] Matalytski M., Kiturko O., Chernaya N., Finding expected incomes in HM-network with priority requests and linear time-dependent intensity of their service, Scientific Research of the Institute of Mathematics and Computer Science Czestochowa University of Technology 2011, 2(10), 179-190.

[3] Chernaya N., Finding the proceeds of the transport logistics system, GrSU, Grodno 2011. 